\title{
Some remarks on $n$-uninorms in IF-sets
}

\author{
Martin Kalina \\ Department of Mathematics and Descriptive Geometry \\ Faculty of Civil Engineering, Slovak University of Technology \\ Radlinského 11, SK-810 05 Bratislava, Slovakia \\ e-mail: martin.kalina@stuba.sk
}

\begin{abstract}
Uninorms and nullnorms are well-known monoidal and monotone operations on the unit interval. Akella [2007] proposed their genaralization to $n$-uninorms. Really, we get both, proper uninorms as well as proper nullnorms as special cases of 2-uninorms. Moreover, proper uninorms as well as proper nullnorms can be characterized as 2-uninorms with some special types of 2-neutral elements. In the present paper, we discuss a classification of 2-uninorms from another point of view as it was done by Akella in 2007 and 2009. Then, we look at 2-uninorms in IF-sets and point out some differences between 2-uninorms on the unit interval and 2-uninorms in IF-sets.
\end{abstract}

Keywords: IF-sets, Nullnorm, Uninorm, $n$-Uninorm.

2010 Mathematics Subject Classification: 03E72, 08A72.

\section{Introduction}

Uninorms were introduced by Yager and Rybalov [21] as a generalization of both $t$-norms and $t$-conorms (for details on $t$-norms and their duals, $t$-conorms, see, e.g., $[13,17]$ ). Since that time, researchers study properties of several distinguished families of uninorms. In [16], Karaçal and Mesiar introduced uninorms in bounded lattices. In [5], Bodjanova and Kalina constructed uninorms in bounded lattices with arbitrarily given underlying $t$-norm and $t$-conorm.

Another generalization of $t$-norms and $t$-conorms, called $t$-operators, was introduced by Mas et al. In [18, 19], Mas et al. studied $t$-operators on finite chains. In 2001, Calvo et al. [6] introduced nullnorms when trying to solve Frank's functional equation [11] where one of the operations in the equation was a uninorm. Afterwards, Mas et al. [20] showed that nullnorms 
and $t$-operators coincide in the unit interval. Karaçal et al. [15] introduced nullnorms in bounded lattices.

Akella [1,2] introduced 2- and $n$-uninorms in the unit interval and gave a characterization of these operations. In this paper, we characterize 2-uninorms (or more general on $n$-uninorms) from the point of view of their two-neutral elements. Particularly, we split the system of all 2-uninorms into 9 (not necessarily disjoint) subclasses. Afterwards, we point out some differences in the structure of 2- (and $n$-) uninorms in IF sets.

Intuitionistic fuzzy sets (also, IF-sets), introduced by Atanassov, are a special type of latticevalued fuzzy sets, introduced by Goguen [12]. Important milestones in the theory of IF-sets, besides the monograph by Atanassov [3], are the papers by Deschrijver [7, 8], and Deschrijver and Kerre [9]. In [7] Deschrijver has shown that there exist $t$-norms which are not representable as a pair of a $t$-norm and a $t$-conorm. In [8] the author has shown that there exist uninorms in IF-sets which are neither conjunctive nor disjunctive. In [9], Deschrijver and Kerre have shown that the theory of IF-sets is equivalent to the theory of interval-valued sets.

A further development of uninorms in IF-sets (or, equivalently, in interval-valued sets) is the paper by Kalina and Král' [14], where the authors have shown that for arbitrary pair (a,e) of incomparable elements of interval-valued sets there exists a uninorm having $\mathbf{a}$ as the annihilator and $\mathbf{e}$ as the neutral element.

\section{Basic definitions and some known facts}

An IF-set [3] can be represented as a special case of $L$-fuzzy set [12], where $L$ is a bounded lattice. Membership grades of an IF-set are elements $\left(x_{1}, x_{2}\right) \in[0,1]^{2}$ such that $x_{1}+x_{2} \leq 1$. The set of all IF-membership grades will be denoted by $L^{*}$. For arbitrary $\left(x_{1}, x_{2}\right),\left(y_{1}, y_{2}\right) \in L^{*}$ the following holds

$$
\left(x_{1}, x_{2}\right) \leq_{L^{*}}\left(y_{1}, y_{2}\right) \Leftrightarrow x_{1} \leq x_{2} \& y_{1} \geq y_{2} .
$$

Thus, the least and the greatest elements of $L^{*}$ are $\mathbf{0}=(0,1), \mathbf{1}=(1,0)$, respectively. We will write these values in bold letters to distinguish them from the real numbers 0 and 1.

Following the notation introduced in [4], we will write $x \| y$ if $x, y \in L^{*}$ are incomparable. For $x \in L^{*}$ we denote $\|_{x}=\left\{z \in L^{*} ; z \| x\right\}$.

Definition 1 ([21]). An associative, commutative and monotone operation $U:[0,1]^{2} \rightarrow[0,1]$ is said to be a uninorm if it has a neutral element $e \in[0,1]$

A uninorm $U$ has an annihilator $a=U(0,1)$, where $a \in\{0,1\}$.

Definition 2 ([10]). A uninorm $U$ is said to be conjunctive if $U(0,1)=0$, and $U$ is called disjunctive if $U(0,1)=1$.

Lemma 1 ([10]). A uninorm $U$ is a t-norm whenever its neutral element is $e=1$. In that case the annihilator of $U$ is $a=0$.

$U$ is a t-conorm whenever its neutral element is $e=0$. In that case the annihilator of $U$ is $a=1$. 
Lemma 2 ([10]). Let $U$ be a uninorm, $e \in] 0,1[$ be its neutral element. Then

$$
T_{U}(x, y)=\frac{U(e x, e y)}{e}, \quad S_{U}(x, y)=\frac{U(e+(1-e) x, e+(1-e) y)-e}{1-e},
$$

are a t-norm and a t-conorm, respectively.

The operations $T_{U}$ and $S_{U}$ from Lemma 2 are called the underlying t-norm and the underlying t-conorm, respectively.

Definition 3 ([6]). An associative, commutative and monotone operation $V:[0,1]^{2} \rightarrow[0,1]$ is said to be a nullnorm if there exists an element $a \in[0,1]$ such that

(1b) $V(0, x)=x$ for all $x \in[0, a]$,

(2b) $V(1, x)=x$ for all $x \in[a, 1]$.

Lemma 3 ([6]). Let $V$ be a nullnorm and $a \in[0,1]$ be such that

(1b) $V(0, x)=x$ for all $x \in[0, a]$,

(2b) $V(1, x)=x$ for all $x \in[a, 1]$.

Then $a$ is the annihilator of $V$.

Similarly like for uninorm $U$, also for nullnorm $V$ there exist its undrlying $t$-norm $V_{T}$ and $t$-conorm $S_{T}$ given by, respectively,

$$
V_{T}(x, y)=\frac{V(a+(1-a) x, a+(1-a) y)-a}{1-a}, \quad V_{S}(x, y)=\frac{V(a x, a y)}{a}
$$

for $a \in] 0,1[$.

Definition 4 ([1]). Let $F:[0,1]^{2} \rightarrow[0,1]$ be a commutative operation. Then $\left\{e_{1}, e_{2}\right\}_{z}$ is called a 2-neutral element of $F$ if $F\left(e_{1}, x\right)=x$ for all $x \in[0, z]$ and $V\left(e_{2}, x\right)=x$ for all $x \in[z, 1]$, where $0<z<1$ and $e_{1} \in[0, z], e_{2} \in[z, 1]$.

Definition 5 ([1]). Let $F:[0,1]^{2} \rightarrow[0,1]$ be a monotone, commutative and associative operation that has a 2-neutral element $\left\{e_{1}, e_{2}\right\}_{z}$.

Lemma 4 ([1]). Let $F$ be a 2-uninorm whose 2-neutral element is $\left\{e_{1}, e_{2}\right\}_{z}$. Then

$$
U_{1}(x, y)=\frac{F(z x, z y)}{z}, \quad U_{2}(x, y)=\frac{F(z+(1-z) x, z+(1-z) y)-z}{1-z}
$$

are uninorms whose neutral elements are $\tilde{e}_{1}=\frac{e_{1}}{z}$ and $\tilde{e}_{2}=\frac{e_{2}-z}{1-z}$, respectively.

Definition 6 ([1]). Let $F:[0,1]^{2} \rightarrow[0,1]$ be a commutative operation and $0=z_{0}<z_{1}<z_{2}<$ $\cdots<z_{n-1}<z_{n}=1$. Then $\left\{e_{1}, e_{2}, \ldots, e_{n}\right\}_{\left(z_{1}, z_{2}, \ldots, z_{n-1}\right)}$ is called an n-neutral element of $F$ if for all $i \in\{1,2, \ldots, n\}$ we have $e_{i} \in\left[z_{i-1}, z_{i}\right]$.

Definition 7 ([1]). An associative, commutative and monotone operation $F:[0,1]^{2} \rightarrow[0,1]$ will be called n-uninorm if it has an n-neutral element $\left\{e_{1}, e_{2}, \ldots, e_{n}\right\}_{\left(z_{1}, z_{2}, \ldots, z_{n-1}\right)}$. 


\section{Characterization and classes of 2-uninorms and a generalization to $n$-uninorms}

Let us consider proper uninorms and proper nullnorms as 1-uninorms. Then we adopt the the following definition:

Definition 8. Let $F_{n}$ be an n-uninorm for $n>1$. We say that $F_{n}$ is a proper $n$-uninorm if $F_{n}$ is not an $(n-1)$-uninorm.

For a proper 2-uninorm $F$, the operations $U_{1}$ and $U_{2}$ given by equality (1), will be called the lower and the upper underlying uninorm, respectively.

Let us a look at 2-neutral elements. For a given $0<z<1$, there are 9 possibilities how to set a 2-neutral element $\left\{e_{1}, e_{2}\right\}_{z}$. Namely,

$$
e_{1}\left\{\begin{array} { l } 
{ = 0 , } \\
{ \in ] 0 , z [ , \quad e _ { 2 } } \\
{ z , }
\end{array} \left\{\begin{array}{l}
=z, \\
\in] z, 1[, \\
1 .
\end{array}\right.\right.
$$

As a corollary to Lemma 1 we get the following

Corollary 1. Let $F$ be a 2-uninorm whose 2-neutral element is $\left\{e_{1}, e_{2}\right\}_{z}$ for $\left.z \in\right] 0,1[$. Set $U_{1}(x, y)=\frac{F(z x, z y)}{z}$ and $U_{2}(x, y)=\frac{F(z+(1-z) x, z+(1-z) y)-z}{1-z}$ Then

(a) $U_{1}$ is a t-norm if $e_{1}=z, U_{2}$ is a t-norm if $e_{2}=1$;

(b) $U_{1}$ is a proper uninorm if $\left.e_{1} \in\right] 0, z\left[\right.$, $U_{2}$ is a proper uninorm if $\left.e_{2} \in\right] z, 1[$;

(c) $U_{1}$ is a t-conorm if $e_{1}=0, U_{2}$ is a t-conorm if $e_{2}=z$.

Let us check all 9 possibilities of setting a 2-neutral element.

Lemma 5. Let $F$ be a 2-uninorm whose 2-neutral element is $\{z, 1\}_{z}\left(\{0, z\}_{z}\right)$ for $0<z<1$. Then $F$ is a t-norm (t-conorm) that is the ordinal sum of two t-norms $F=\left(\left\langle T_{1}, 0, z\right\rangle,\left\langle T_{2}, z, 1\right\rangle\right)$ (of two t-conorms $F=\left(\left\langle S_{1}, 0, z\right\rangle,\left\langle S_{2}, z, 1\right\rangle\right)$ ).

Proof. We will prove only the $t$-norm case.

As the first step, let us prove that $F(0,1)=0$. Since $\{z, 1\}_{z}$ is the 2-neutral element of $F$, we have by associativity

$$
F(0,1)=F(F(0, z), 1)=F(0, F(z, 1))=F(0, z)=0 .
$$

Monotonicity of $F$ implies that 0 is the annihilator of $F$.

As the second step, we prove that 1 is the neutral element of $F$. Since we know that 1 is the partial neutral element of $F$ in the interval $[z, 1]$. Let $x \in[0, z]$.

$$
F(x, 1)=F(F(x, z), 1)=F(x, F(z, 1))=F(x, z)=x .
$$

The proof is completed. 
Lemma 6. Let $F$ be a 2-uninorm whose 2-neutral element is $\{z\}_{z}\left(\left\{e_{1}, z\right\}_{z},\left\{z, e_{2}\right\}_{z}\right)$ for $0<z<1$ and $0<e_{1}<z, z<e_{2}<1$. Then $F$ is a uninorm whose neutral element is $z$ ( $e_{1}$ and the underlying $t$-conorm $S$ is the ordinal sum of two $t$-conorms $S=\left(\left\langle S_{1}, e_{1}, z\right\rangle,\left\langle S_{2}, z, 1\right\rangle\right)$, $e_{2}$ and the underlying $t$-norm $T$ is the ordinal sum of two $t$-norms $\left.T=\left(\left\langle T_{1}, 0, z\right\rangle,\left\langle T_{2}, z, e_{2}\right\rangle\right)\right)$.

Proof. In the case that $\{z\}_{z}$, we have that $z$ is a partial neutral element in the interval $[0, z]$ as well as in the interval $[z, 1]$, i.e., $z$ is the neutral element of $F$. Hence, directly by Definition 1 we get that $F$ is a uninorm with the neutral element $z$.

In the case that $\left\{e_{1}, z\right\}_{z}$ is the 2-neutral element of $F$, we get applying Lemma 5 to the interval $\left[e_{1}, 1\right]$ that $S=\left(\left\langle S_{1}, e_{1}, z\right\rangle,\left\langle S_{2}, z, 1\right\rangle\right)$ is a $t$-conorm which is the underlying operation of $F$. The rest of the proof is due to Definition 1.

Dually we could prove the case when $\left\{z, e_{2}\right\}_{z}$ is the 2-neutral element of $F$.

Lemma 7. Let $F$ be a 2-uninorm whose 2-neutral element is $\{0,1\}_{z}$ for $0<z<1$. Then, $F$ is a nullnorm and $z$ is its annihilator.

Proof. The fact that $F$ is a nullnorm with the annihilator $z$ is directly due to Definition 3.

The remaining three cases lead to proper 2-uninorms.

Lemma 8. Let $F$ be a 2-uninorm whose 2-neutral element is $\left\{e_{1}, e_{2}\right\}_{z}$ for $0<e_{1}<z<e_{2}<1$. Then $F$ is a proper 2-uninorm.

We omit the proof of this lemma since the assertion is obvious.

Lemma 9. Let $F$ be a 2-uninorm whose 2-neutral element is $\{e, 1\}_{z}$ for $0<e<z<1$. Then $F$ is a proper 2-uninorm whose upper underlying uninorm is reduced to a t-norm.

Proof. The fact that the upper underlying uninorm is reduced to a $t$-norm is due to Lemma 5 . The rest of the proof is obvious.

Lemma 10. Let $F$ be a 2-uninorm whose 2-neutral element is $\{0, e\}_{z}$ for $0<z<e<1$. Then $F$ is a proper 2-uninorm whose lower underlying uninorm is reduced to a t-conorm.

The assertion of Lemma 10 is a dual case of Lemma 9. That is why the proof is omitted. Generalizing Lemma 6, we get the following

Proposition 1. For $n \geq 2$, let $F$ be a proper $n$-uninorm where $\left\{e_{1}, e_{2}, \ldots, e_{n}\right\}_{\left(z_{1}, z_{2}, \ldots, z_{n-1}\right)}$ is its $n$-neutral element. Then there exists $1 \leq i \leq n$ such that $z_{i-1}<e_{i}<z_{i}$ and moreover, $F$ is an $(n+1)$-uninorm whose $(n+1)$-neutral element is $\left\{e_{1}, e_{2}, \ldots, e_{n}\right\}_{\left(z_{1}, z_{2}, \ldots, e_{i}, z_{i}, \ldots, z_{n-1}\right)}$.

Proof. We have to prove two items for $n \geq 2$ :

1) There exists $i$ such that $z_{i-1}<e_{i}<z_{i}$,

2) $\left\{e_{1}, e_{2}, \ldots, e_{n}\right\}_{\left(z_{1}, z_{2}, \ldots, e_{i}, z_{i}, \ldots, z_{n-1}\right)}$ is an $(n+1)$-neutral element of $F$. 
To prove item 1), it is enough to realize that, for $n \geq 2$, if there were no $i$ such that $z_{i-1}<e_{i}<z_{i}$, the operation $F$ would have diagonal blocks either $\left(T_{1}, S_{1}, T_{2}, S_{2}, \ldots\right)$ or $\left(S_{1}, T_{1}, S_{2}, T_{2}, \ldots\right)$, where $T_{1}, T_{2}$ are $t$-norms, and $S_{1}, S_{2}$ are $t$-conorms. In each of these two cases the $n$-neutral element could be reduced to the $(n-1)$-neutral element, since in the first case $e_{1}=e_{2}$ and in the second case $e_{2}=e_{3}$ either $\left\{e_{2}, \ldots, e_{n}\right\}_{\left(z_{2}, \ldots, e_{i}, z_{i}, \ldots, z_{n-1}\right)}$ or $\left\{e_{1}, \ldots, e_{n}\right\}_{\left(z_{1}, \ldots, e_{i}, z_{i}, \ldots, z_{n-1}\right)}$, respectively. This proves the item 1 ) for $n \geq 3$. For $n=2$ the statement is due to Lemmas 8,9 and 10.

Item 2) is a direct consequence of item 1).

\section{2-uninorms on IF-sets}

$\left(L^{*}, \leq_{L^{*}}\right)$ is a bounded lattice with incomparable elements. The incomparability of some elements will be crucial in our considerations.

Example 1. On the bounded lattice $\left(L^{*}, \wedge, \vee, \mathbf{0}, \mathbf{1}\right), T_{\wedge}\left(z_{1}, z_{2}\right)=z_{1} \wedge z_{2}$ is the greatest $t$-norm. When we choose an arbitrary element $x \notin\{\mathbf{0}, \mathbf{1}\}, T_{\wedge}$ can be considered as the ordinal sum $t$-norm $\left(\left\langle T_{\wedge}, \mathbf{0}, x\right\rangle,\left\langle T_{\wedge}, x, \mathbf{1}\right\rangle\right)$.

On the other hand, since $\|_{x} \neq \emptyset$, we can define

$$
\tilde{T}_{\wedge}\left(z_{1}, z_{2}\right)= \begin{cases}z_{1} \wedge z_{2} & \text { for }\left(z_{1}, z_{2}\right) \in([\mathbf{0}, x] \cup[x, \mathbf{1}])^{2}, \\ z_{2} & \text { for } z_{1} \in \|_{x}, z_{2} \in[\mathbf{0}, x] \\ z_{1} & \text { for } z_{1} \in[\mathbf{0}, x], z_{1} \in \|_{x} \\ x & \text { otherwise. }\end{cases}
$$

Hence, $\tilde{T}_{\wedge}$ is not a $t$-norm, but $\{x, 1\}_{x}$ is a 2 -neutral element of $\tilde{T}_{\wedge}$. This means that $\tilde{T}_{\wedge}$ is a proper 2-uninorm.

Example 2. Let $x \notin\{0,1\}$ be an element in $L^{*}$.

$$
\tilde{U}\left(z_{1}, z_{2}\right)= \begin{cases}z_{1} \wedge z_{2} & \text { for }\left(z_{1}, z_{2}\right) \in[\mathbf{0}, x]^{2}, \\ z_{1} & \text { for } z_{1} \in[\mathbf{0}, x] \text { and } z_{2} \notin[\mathbf{0}, x], \\ & \text { and for } z_{1} \in[x, \mathbf{1}] \text { and } z_{2} \in \|_{x}, \\ z_{2} & \text { for } z_{2} \in[\mathbf{0}, x] \text { and } z_{1} \notin[\mathbf{0}, x], \\ & \text { and for } z_{2} \in[x, \mathbf{1}] \text { and } z_{1} \in \|_{x}, \\ z_{1} \vee z_{2} & \text { for }\left(z_{1}, z_{2}\right) \in[x, \mathbf{1}]^{2} \\ x & \text { otherwise. }\end{cases}
$$

The operation $\tilde{U}$ is restricted to $[\mathbf{0}, x] \cup[x, \mathbf{1}]$, if $\tilde{U}$ has no neutral element on the whole $L^{*}$, i.e., it is not a uninorm. On the other hand, $\{x\}_{x}$ is a 2-neutral element, hence $\tilde{U}$ is a proper 2-uninorm. 
Example 3. Let $x \notin\{\mathbf{0}, \mathbf{1}\}$ be an element in $L^{*}$.

$$
V\left(z_{1}, z_{2}\right)= \begin{cases}z_{1} \vee z_{2} & \text { for }\left(z_{1}, z_{2}\right) \in[\mathbf{0}, x]^{2} \\ z_{1} \wedge z_{2} & \text { for }\left(z_{1}, z_{2}\right) \in[x, \mathbf{1}]^{2} \\ x & \text { otherwise }\end{cases}
$$

$V$ is a nullnorm whose annihilator is $x$. In this case, if we are looking for a modification $\tilde{V}$ of $V$ in such a way that $\tilde{V}$ is reduced to $[\mathbf{0}, x] \cup[x, \mathbf{1}]$, but $\tilde{V}$ is not a nullnorm, we will not succeed. Really, we have that $V(\mathbf{1}, \mathbf{0})=x$ and hence also $\tilde{V}(\mathbf{1}, \mathbf{0})=x$ and this implies that $x$ is the annihilator of $\tilde{V}$.

Remark 1. Dually to the operation $\tilde{T}_{\wedge}$ introduced by (2), we can define on $L^{*}$ an operation $\tilde{S}_{\vee}$ starting from the $t$-conorm $S_{\vee}\left(z_{1}, z_{2}\right)=z_{1} \vee z_{2}$ and an element $x \notin\{\mathbf{0}, \mathbf{1}\}$. This means that, unlike the situation with the operations in the unit interval, an arbitrary form of the 2-neutral element, except of the case when $\{\mathbf{0}, \mathbf{1}\}_{x}$ is the 2-neutral element, may lead to proper 2-uninorms.

As a corollary to the above considerations in Examples 1-3, we get the following proposition.

Proposition 2. For arbitrary $n \geq 2$ there exists a proper $n$-uninorm $F: L^{*} \times L^{*} \rightarrow L^{*}$ such that $F$ has no $(n+1)$-neutral element, i.e., $F$ is not an $(n+1)$-uninorm.

Proof. It is enough to modify the construction in Example 1. For arbitrary $n \geq 2$, let us choose $\mathbf{0}=\zeta_{0}<\zeta_{1}<\zeta_{2}<\cdots<\zeta_{n-1}<\zeta_{n}=\mathbf{1}$ and we define an operation $\tilde{T}$ by

$$
\tilde{T}\left(z_{1}, z_{2}\right)= \begin{cases}z_{1} & \text { for } z_{1} \in\left[\zeta_{i-1}, \zeta_{i}\right], i \in\{1,2, \ldots n-1\} \text { and } z_{2} \geq \zeta_{i}, \\ z_{2} & \text { for } z_{2} \in\left[\zeta_{i-1}, \zeta_{i}\right], i \in\{1,2, \ldots n-1\} \text { and } z_{1} \geq \zeta_{i}, \\ \zeta_{i-1} & \text { for } i \in\{1,2, \ldots, n\} \text { and }\left(z_{1}, z_{2}\right) \in\left[\zeta_{i-1},\left.\zeta_{i}\right|^{2},\right. \\ & \text { or }\left(z_{1}, z_{2}\right) \in\left[\zeta_{i-1}, \mathbf{1}\right]^{2} \text { and } z_{1} \|_{\zeta_{i}} \text { or } z_{2} \|_{\zeta_{i}} .\end{cases}
$$

We get that $\left\{\zeta_{1}, \zeta_{2}, \ldots, \zeta_{n}\right\}_{\left(\zeta_{1}, \zeta_{2}, \ldots, \zeta_{n-1}\right)}$ is the $n$-neutral element of $\tilde{T}$ and there exists no $(n+1)$ neutral element of $\tilde{T}$.

\section{Conclusions}

In this paper, we have discussed 2-uninorms in the unit interval and in the $L^{*}$ lattice of IF-membership grades. We have shown that there are substantial differences between 2-uninorms in the unit interval and 2-uninorms in the $L^{*}$ lattice. The results on 2-uninorms we have generalized to $n$-uninorms.

\section{Acknowledgements}

The work on this paper was supported from the VEGA grant agency, grant No. 1/0006/19, and from the APVV grant agency, grant No. 18-0052. 


\section{References}

[1] Akella, P. (2007). Structure of $n$-uninorms, Fuzzy Sets and Systems, 158, 1631-1651.

[2] Akella, P. (2009). C-sets of n-uninorms, Fuzzy Sets and Systems, 160, 1-21.

[3] Atanasov, K.T. (1999). Intuitionistic Fuzzy Sets. Springer, Heidelberg.

[4] Birkhoff, G. (1967). Lattice Theory. American Mathematical Society Colloquium Publishers, Providence, RI.

[5] Bodjanova, S. \& Kalina, M. (2014). Construction of uninorms on bounded lattices. In: IEEE 12th International Symposium on Intelligent Systems and Informatics, SISY 2014, Subotica, $61-66$.

[6] Calvo, T., De Baets, B. \& Fodor, J. (2001). The functional equations of Frank and Alsina for uninorms and nullnorms, Fuzzy Sets and Systems, 120, 385-394.

[7] Deschrijver, G. (2008). A representation of $t$-norms in interval valued L-fuzzy set theory, Fuzzy Sets and Systems, 159, 1597-1618.

[8] Deschrijver, G. (2013). Uninorms which are neither conjunctive nor disjunctive in intervalvalued fuzzy set theory, Information Sciences, 244, 48-59.

[9] Deschrijver, G. \& Kerre, E.E. (2003). On the relationship between some extensions of fuzzy set theory, Fuzzy Sets and Systems, 133(2), 227-235.

[10] Fodor, J., Yager, R.R. \& Rybalov, A. (1997). Structure of uninorms. Int. J uncertainty, Fuzziness and Knowledge-Based Systems, 5, 411-427.

[11] Frank, M. (1979). On the simultaneous associativity of $F(x ; y)$ and $x+y-F(x ; y)$, Aeq. Math., 19, 194-226.

[12] Goguen, J. A. (1967). L-fuzzy sets. Journal of Mathematical Analysis and Applications, $18(1), 145-174$.

[13] Grabisch, M., Marichal, J.L., Mesiar, R., \& Pap, E. (2009). Aggregation Functions, Cambridge University Press, Cambridge.

[14] Kalina, M., \& Král', P. (2016). Uninorms on interval-valued fuzzy sets, In: Carvalho J., Lesot MJ., Kaymak U., Vieira S., Bouchon-Meunier B., Yager R. (eds) Information Processing and Management of Uncertainty in Knowledge-Based Systems. IPMU 2016, Communications in Computer and Information Science, vol 611. Springer, Cham, 522-531.

[15] Karaçal, F., Ince, M. A., \& Mesiar, R. (2015). Nullnorms on bounded lattices. Information Sciences, 325, 227-236

[16] Karaçal, F., \& Mesiar, R. (2015). Uninorms on bounded lattices. Fuzzy Sets and Systems, 261, 33-43. 
[17] Klement, E. P., Mesiar, R., \& Pap, E. (2000). Triangular Norms. Springer, Berlin, Heidelberg.

[18] Mas, M., Mayor, G., \& Torrens, J. (1999). t-operators. Journal of Uncertainty, Fuzziness and Knowledge-Based Systems 7, 31-50.

[19] Mas, M., Mayor, G., \& Torrens, J. (1999). t-operators and uninorms in a finite totally ordered set. Internat. J. Intell. Systems, 14 (9), 909-922.

[20] Mas, M., Mayor, G., \& Torrens, J. (2002). The distributivity condition for uninorms and t-operators. Fuzzy Sets and Systems 128, 209-225.

[21] Yager, R. R., \& Rybalov, A. (1996). Uninorm aggregation operators, Fuzzy Sets and Systems, 80, 111-120. 